09

\title{
Методы возбуждения параметрического резонанса в схеме оптического магнитометрического датчика
}

\author{
(C) А.К. Вершовский, М.В. Петренко \\ Физико-технический институт им. А.Ф. Иофрфе РАН, \\ 194021 Санкт-Петербург, Россия \\ e-mail: antver@mail.ioffe.ru
}

Поступило в Редакцию 7 декабря 2020 г.

В окончательной редакции 7 декабря 2020 r.

Принято к публикации 10 декабря 2020 г.

Проведено экспериментальное сравнение методов модуляции параметров поперечного по отношению к внешнему магнитному полю резонансного излучения накачки в двухлучевой схеме оптического магнитометрического датчика (схеме Белла-Блюма), а также сравнение этих методов со стандартным методом радиочастотного возбуждения магнитного резонанса в условиях сильной лазерной накачки. Показано, что, хотя стандартный метод позволяет добиться большего подавления спин-обменного уширения линии магнитного резонанса светом накачки, схема Белла-Блюма характеризуется преимуществами, позволяющими получать близкие значения чувствительности при модуляции как интенсивности, так и поляризации света накачки; при этом схема Белла-Блюма потенциально характеризуется большим быстродействием, что существенно для задач магнитоэнцефалографии и магниторезонансной томографии сверхслабого поля.

Ключевые слова: оптически детектируемый магнитный резонанс, квантовый магнитометр, магнитоэнцефалография, томография сверхслабого поля.

DOI: $10.21883 /$ JTF.2021.05.50698.337-20

\section{Введение}

Оптические датчики магнитного поля, основанные на эффекте оптически детектируемого магнитного резонанса (МР), в течение нескольких десятилетий характеризуются непревзойденным сочетанием точности, чувствительности и быстродействия [1]. Традиционно эти датчики использовались преимущественно в задачах геологоразведки, однако в последние годы происходит стремительное увеличение востребованности этих устройств в биомедицинских исследованиях, в первую очередь - для использования в магнитоэнцефалографических (МЭГ) комплексах [2] и системах магнитнорезонансной томографии (МРТ) сверхслабого поля [3]. Соответственно изменяются требования к этим устройствам: и в системах МЭГ, и в системах МРТ измеряются только переменные магнитные поля, следовательно, смягчаются требования к точности датчика; одновременно возрастают требования к его чувствительности, компактности и быстродействию, а также к способности датчиков функционировать в составе многоканальной системы, не создавая помех друг другу.

Способ решения последней проблемы был предложен в [4] - вместо стандартного возбуждения МР резонансным радиочастотным полем в ансамбле атомов, магнитные моменты которых предварительно ориентированы вдоль магнитного поля циркулярно поляризованным лучом оптической накачки (далее - „классическая схема“), было использовано возбуждение МР поперечным по отношению к магнитному полю лучом накачки, модулированным на частоте прецессии этих моментов, т.е. ларморовской частоте (далее - схема Белла-Блюма); это позволило избежать возникновения взаимных радиочастотных помех при совместной работе в схеме МЭГ.

Значительную трудность представляет собой одновременное увеличение чувствительности, компактности и быстродействия, поскольку предельная чувствительность квантовых датчиков увеличивается пропорционально корню из их объема и падает пропорционально ширине MP [1], а быстродействие датчика определяется шириной МР. Очевидным путем к решению задачи компактизации является отказ от ячеек с антирелаксационным покрытием с одновременным повышением концентрации рабочих атомов в ячейке - что, в свою очередь, ведет к увеличению спин-обменного уширения линии МР.

Наиболее радикально проблема повышения чувствительности решена в датчиках на компактных газовых ячейках, работающих в режиме SERF (spin-exchange relaxation free - подавление спин-обменного уширения линии МР в нулевом магнитном поле) [5,6]; однако область применения этих датчиков ограничена полями, не превышающими несколько десятков или сотен нанотесла.

Несколько худших значений чувствительности достигают схемы, работающие в режиме частичного светового подавления спин-обменного уширения [7,8], реализующегося в ненулевых полях при сильной лазерной накачке циркулярно поляризованным светом $D_{1}$ линии: по мере того, как атомы сосредотачиваются на единственном не взаимодействующем со светом магнитном 
уровне $|F=I+1 / 2, m F=F\rangle$, где $I-$ момент ядра (для Cs $I=7 / 2$ ), уменьшается количество их возможных партнеров по спин-обмену (так называемое stretched state - вытянутое состояние [9]), и падает скорость спин-обмена. К настоящему времени в таких схемах реализованы значения чувствительности, позволяющие успешно использовать их в системах МЭГ $[8,10]$.

Наиболее перспективными среди схем датчиков ненулевого поля показали себя схемы, использующие оптическую накачку, настроенную в резонанс с переходами со сверхтонкого уровня $F=I-1 / 2$ основного состояния $S_{1 / 2}$ на ближайшие возбужденные уровни $F=I-1 / 2 P_{1 / 2}[7,11]$. Детектирование МР на переходе $F=I+1 / 2, m_{F}=F \leftrightarrow F-1$ основного состояния осуществляется на ларморовской частоте (так называемая $M_{X}$-схема) по повороту поляризации нерезонансного линейно поляризованного поперечного пробного луча, отстроенного от соответствующей оптической линии поглощения вниз по частоте на несколько ширин оптической линии.

В схеме Белла-Блюма используется так называемый параметрический резонанс, возбуждаемый посредством модуляции различных параметров оптической накачки: интенсивности, частоты, поляризации. Параметры МР при различных схемах модуляции были подробно исследованы в [12]. В настоящей работе мы проводим сравнение методов модуляции интенсивности и поляризации излучения накачки и сравниваем их с „классическим“ методом возбуждения МР. Если не рассматривать световые сдвиги МР, то модуляция отстройки частоты излучения накачки от оптической линии поглощения физически эквивалентна изменению модуляции интенсивности накачки, и далее отдельно рассматриваться не будет.

\section{1. Эксперимент}

Экспериментальная установка, на которой производились измерения, описана в $[10,13]$. МР возбуждался в кубических ячейках размером $5 \times 5 \times 5$ и $8 \times 8 \times 8 \mathrm{~mm}$ производства компании VitaWave. Ячейки содержали насыщенные пары Cs и азот под давлением 100 Torr. Исследуемая ячейка размещалась в центральной области многослойного магнитного экрана, в котором поддерживалась индукция магнитного поля $\sim 12 \mu \mathrm{T}$. По сравнению с [10] установка была дополнена съемным блоком, включающим линейный поляризатор и четвертьволновую пластину и позволяющим использовать электрооптический модулятор (ЕОМ) для модуляции интенсивности накачки (рис. 1). В режиме модуляции интенсивности циркулярная поляризация накачки поддерживалась постоянной, в режиме модуляции поляризации эллиптичность поляризации излучения накачки варьировалась от 1 до +1 (т.е. от левой до правой циркулярной поляризации, в других принятых в оптике

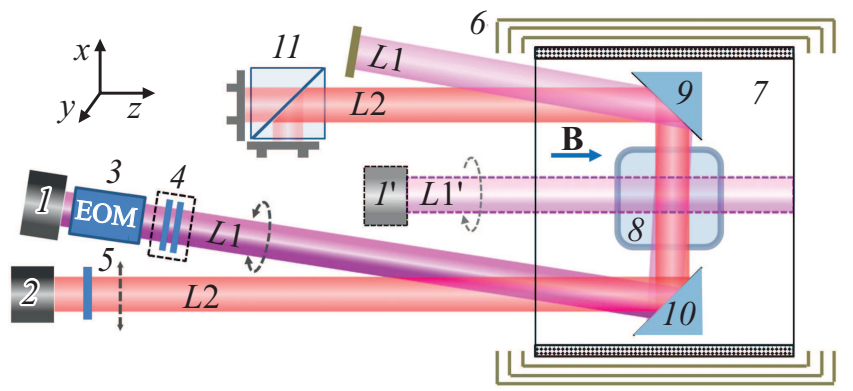

Рис. 1. Упрощенная схема квантового датчика в схеме Белла-Блюма: 1 - ввод излучения накачки, $2-$ ввод пробного излучения, $L 1$ - луч оптической накачки, $L 2-$ пробный луч, 3 - электрооптический (циркулярный) модулятор поляризации, 4 - съемный блок, включающий линейный поляризатор и четвертьволновую пластину, 5 - линейный поляризатор, 6 - магнитный экран, 7 - соленоид, 8 газовая ячейка с парами $\mathrm{Cs}, 9,10$ - зеркала, 11 - балансный фотоприемник. $1^{\prime}$ и $L 1^{\prime}-$ ввод излучения накачки и луч оптической накачки в „классической“ схеме (катушки, создающие резонансное радиочастотное поле, не показаны).

обозначениях - от $-45^{\circ}$ до $+45^{\circ}$ ) при постоянной интенсивности.

\section{2. Обсуждение}

Мы провели сравнение трех типов модуляции (симметричной прямоугольной, синусоидальной и симметричной треугольной) поляризации луча накачки; на рис. 2 приведены результаты измерений параметров MP от глубины модуляции (входное напряжения ЕOM $0 \mathrm{~V}$ соответствует линейной поляризации накачки, $\pm 20 \mathrm{~V}-$ круговой поляризации, показатель эллиптичности поляризации излучения при изменении входного напряжения ЕОМ составляет $2.25 \mathrm{deg} / \mathrm{V})$. В условиях, при которых производились измерения $\left(T=100^{\circ} \mathrm{C}\right.$, максимальная интенсивность лучей накачки и детектирования), световое подавление спин-обменного уширения МР почти полностью маскируется световым уширением. Слабое (лежащее в пределах погрешности измерений) подавление уширения при увеличении амплитуды входного напряжения ЕОМ прослеживается только в интервале $1-4 \mathrm{Vpp}$ (рис. 2,a).

Приведенные на рис. 2, а зависимости характеризуются сходным поведением при росте амплитуды модулирующего сигнала, но отличаются скоростью изменения, т. е. масштабом по горизонтальной оси. То же относится и к зависимостям на рис. $2, b$. На рис. 3 приведены те же зависимости, масштабированные по оси абсцисс таким образом, чтобы обеспечить максимальное совпадение результатов трех серий (значения коэффициента масштабирования $k_{x}$ приведены на графике).

Сопоставление показало, что крутизна линии МР определяется амплитудой первой гармоники спектра модулирующего сигнала (рис. $3, b$ ). Это совершенно 


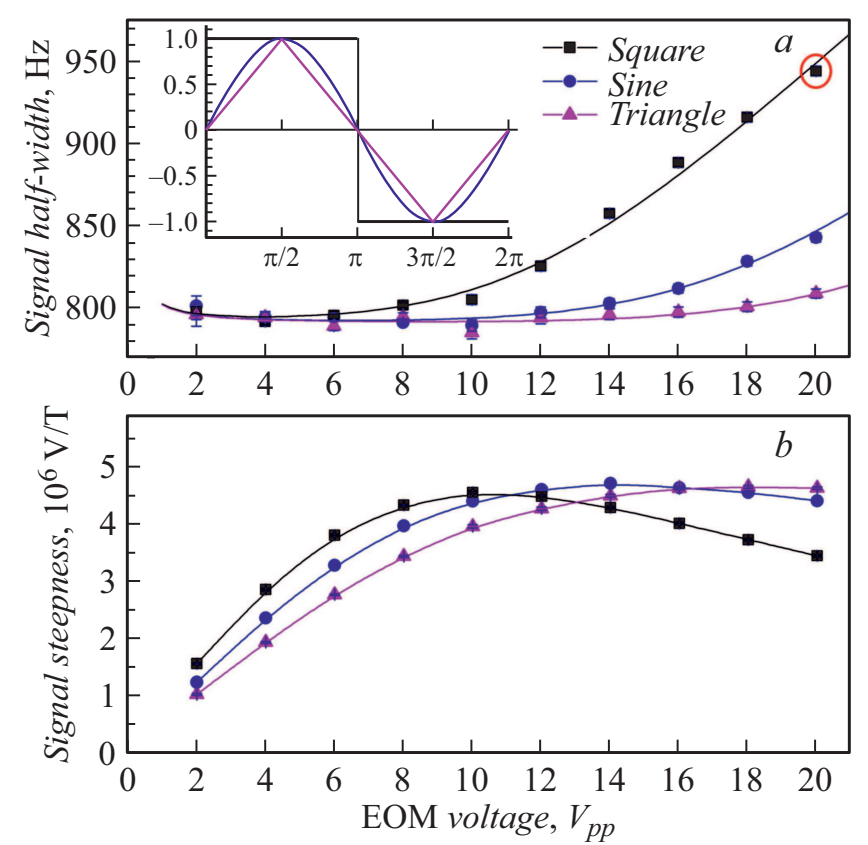

Рис. 2. Сравнение параметров МР при модуляции поляризации при сильной $(5.6 \mathrm{~mW})$ мощности пробного луча и температуре $100^{\circ} \mathrm{C}$. На врезке - формы модулирующих функций.

естественно, поскольку все остальные гармоники частоты модуляции лежат далеко за пределами ширины линии МР и не дают вклада в накачку.

В то же самое время оказалось, что уширение линии МР преимущественно определяется нормированной площадью модулирующей функции, т. е. интегралом от ее модуля за период (рис. 3,a).

Определим уширение линии МР модуляцией как превышение текущей ширины линии над минимальной шириной на той же зависимости. Тогда величина уширения в оптимуме, определенном по критерию максимальной крутизны, для синусоидальной модуляции не превышает $10 \mathrm{~Hz}$, или $\sim 1 \%$ от ширины резонанса. В случае прямоугольной модуляции можно также ввести асимметрию модуляции и варьировать зависимость уширения линии MP от коэффициента $k_{f}$ заполнения модулирующей функции $\left(k_{f}=1-k_{d}\right.$, где $k_{d}$ - скважность модуляции). Такая зависимость приведена на рис. 4; на врезке показаны амплитуды первых гармоник спектра модулирующего сигнала при различных значениях $k_{f}$. Данные рис. 4 подтверждают, что уширение резонанса лишь частично определяется амплитудой первой гармоники модуляции.

Далее были исследованы параметры МР в широком диапазоне температур ячейки и интенсивностей излучения накачки; параметры MP в „классической“ схеме исследовались также в широком диапазоне амплитуд резонансного радиополя, что позволило в каждой точке по температуре и интенсивности произвести экстраполяцию ширин к нулю амплитуды радиополя. Интенсивность детектирующего (пробного) излучения была выбрана заведомо небольшой $(0.5 \mathrm{~mW})$, чтобы при расстройке $\sim 30 \mathrm{GHz}$ она не оказывала заметного уширяющего влияния на ширину МР.

На экспериментально измеренных зависимостях ширин МР от интенсивности накачки, приведенных на

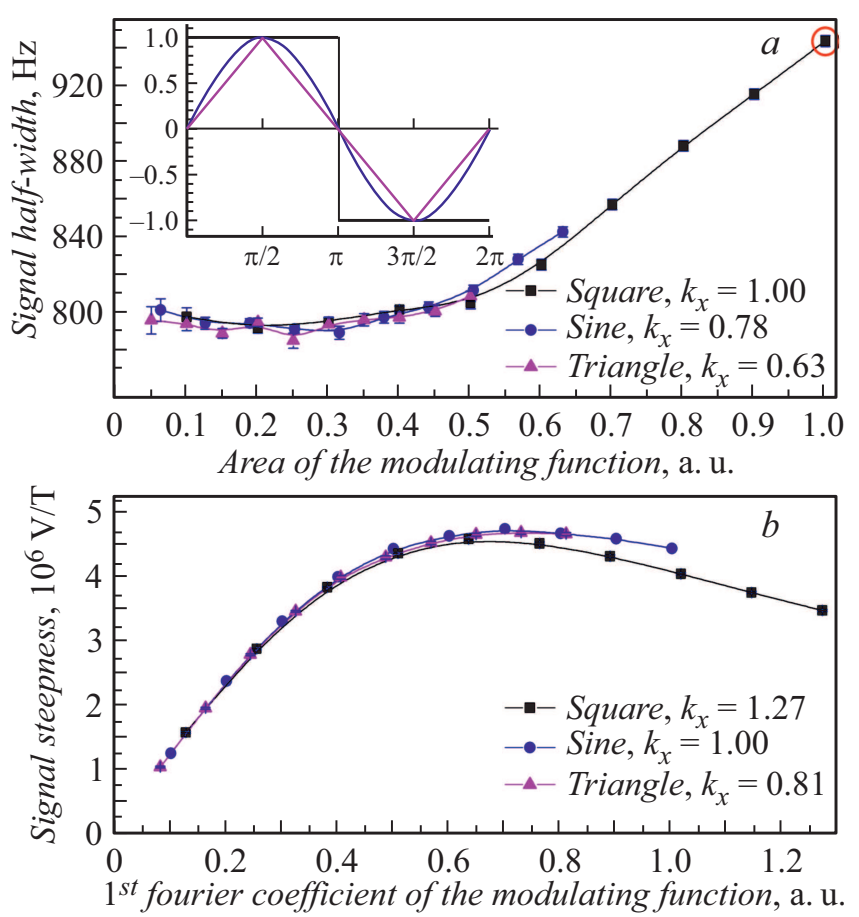

Рис. 3. Сравнение полуширины линии МР $(a)$ и крутизны линии МР $(b)$ для трех форм модулирующих функций при модуляции поляризации; по оси абсцисс отложены: $a$ нормированные значения площади модулирующей функции, $b$ - нормированные амплитуды первых гармоник спектра модулирующих функций. На врезке показаны формы спектров модулирующих функций.

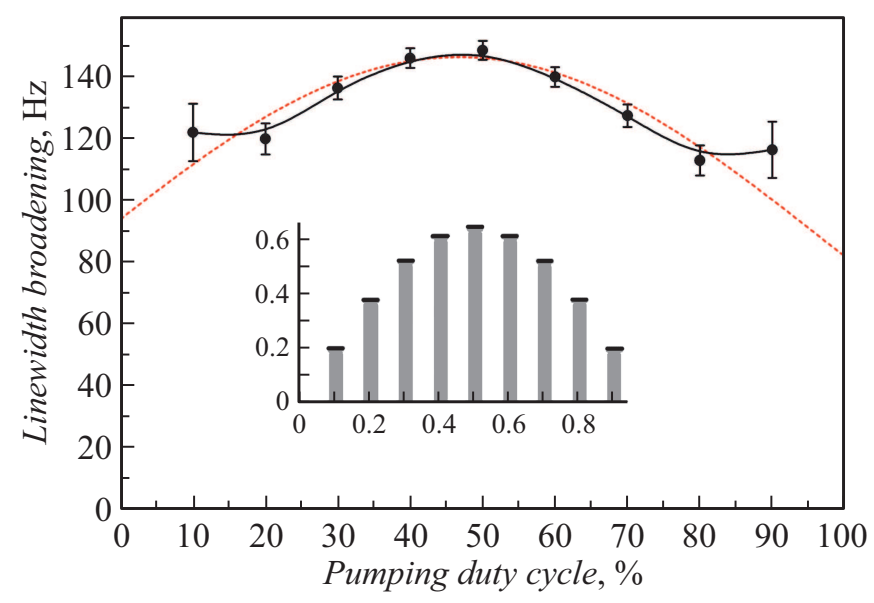

Рис. 4. Зависимость уширения МР модуляцией от коэффициента заполнения $k_{f}$ прямоугольной модуляции поляризации накачки при максимальном индексе модуляции (точка, обведенная кружком на рис. 2, $a, 3, a)$. Пунктир - аппроксимация синусоидальной зависимостью. На врезке - амплитуды первых гармоник спектра модулирующего сигнала в зависимости от $k_{f}$. 


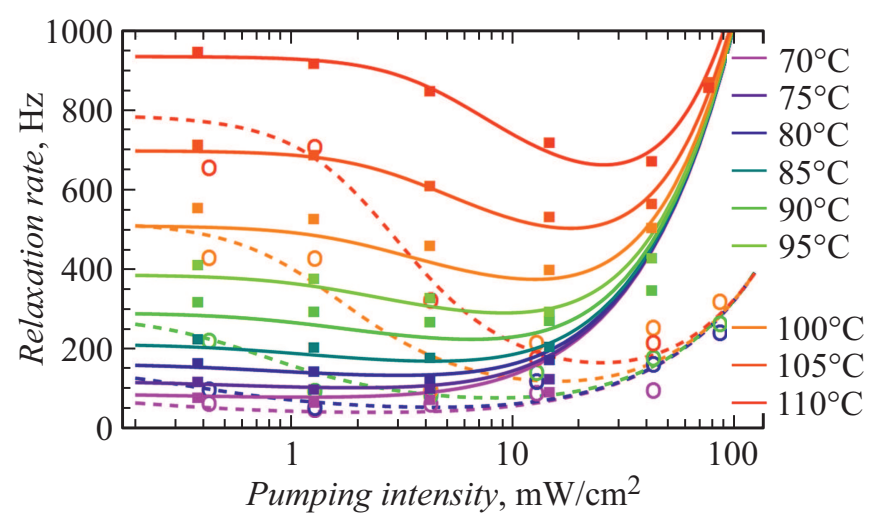

Рис. 5. Экспериментально измеренные ширины резонансов в „классической“ схеме (незалитые символы+пунктир, $T=70$, $\left.80,90,100,110^{\circ} \mathrm{C}\right)$ и схеме Белла-Блюма при модуляции поляризации (залитые символы + сплошные линии, $T=70$, $\left.75,80,85,90,95,100,105,110^{\circ} \mathrm{C}\right)$.

рис. 5, четко прослеживаются три области: 1) область слабых интенсивностей, в которой ширина резонанса преимущественно определяется спин-обменным уширением; 2) область средних интенсивностей, в которой происходит эффективное световое подавление спинобменного уширения; 3) область высоких интенсивностей, в которой доминирует уширение резонанса светом накачки.

Видно, что в области средних интенсивностей ширина резонанса в схеме Белла-Блюма в 3.5-4 раза превышает ширину в „классической“ схеме. Это в значительной степени компенсируется двумя факторами: во-первых, отсутствием в схеме Белла-Блюма резонансного радиополя, которое в „классической“ схеме, оптимизированной по критерию максимума крутизны MP, дополнительно уширяет MP в $\sqrt{ } 2$ раз, во-вторых, тем, что в схеме Белла-Блюма магнитные моменты прецессируют в плоскости, перпендикулярной магнитному полю, и их проекция на пробный луч оказывается в $\sqrt{ } 2$ раз больше, чем в „классической“ схеме, где при оптимальной амплитуде радиополя моменты отклонены от вектора магнитного поля на $45^{\circ}$. Следовательно, схема Белла-Блюма преимущественно должна применяться в задачах, не требующих абсолютной точности, но требующих высокого быстродействия - например, в системах МЭГ и МРТ сверхслабого поля.

Объяснить меньшую степень подавления светового уширения в схеме Белла-Блюма можно, сравнив механизмы накачки в этих двух схемах (рис. 6).

Накачка в „классической“ схеме осуществляется непрерывно вдоль вектора магнитного поля $\mathbf{B}_{0} \| z$. Резонансное радиополе $B_{1} \cdot \cos \left(\omega_{1} \cdot t\right)$ отклоняет выстроенные вдоль оси $z$ моменты, как это показано на рис. $6, a$, и собирает их в один коллективный момент, прецессирующий под постоянным углом к оси $z$ (мы здесь пренебрегаем разбросом проекций моментов, связанным с релаксацией). Если перейти в систему координат, вращающуюся вокруг оси $z$ с частотой $\omega_{1} \approx \omega_{0}=\gamma \cdot B_{0}$, в которой единственным действующим полем является постоянное в этой системе координат поле $\mathbf{B}_{1} \| \mathbf{y}^{\prime}$, то мы увидим, что прецессия вокруг $\mathbf{B}_{1}$ в каждый момент времени отклоняет магнитные моменты в одном и том же направлении, обеспечивая тем самым высокую степень когерентности суммарного момента, являющуюся основным условием „вытянутого“ состояния (рис. $6, b$ ).

В схеме Белла-Блюма фазирование веера магнитных моментов осуществляется посредством модуляции накачки, и величина размытия суммарного момента по фазе зависит от типа модуляции; в частности, можно ожидать, что при модуляции интенсивности синусоидальная модуляция (рис. 6,c) или модуляция меандром приведет к большему размытию и, следовательно, к меньшему подавлению спин-обменного уширения, чем импульсная накачка с высокой скважностью, т.е. малым коэффициентом заполнения (рис. $6, d$ ).

Результаты сравнения параметров МР при синусоидальной модуляции интенсивности и поляризации приведены на рис. 7. Видно, что значения ширин и крутизны МР в этих двух режимах практически совпадают, несмотря на то, что в режиме модуляции интенсивности эффективная накачка осуществляется один раз в период, a в режиме модуляции поляризации (в котором при постоянной интенсивности накачки знак циркулярной поляризации меняется на противоположный) - два раза за период. Отметим, что для достижения той же средней по времени интенсивности накачки в режиме модуляции поляризации требуется вдвое меньшая интенсивность лазерного излучения, чем в режиме модуляции интенсивности.

Если верны приведенные выше рассуждения (рис. 6, d), „вытянутое состояние“ может быть получено посредством модуляции интенсивности накачки с высокой скважностью (т. е. с малым коэффициентом заполнения $k_{f}$ ). На рис. 8 приведены результаты измерения параметров МР при изменении коэффициента заполнения модуляции интенсивности накачки. Измерения проводились в двух режимах - при постоянной интенсивности на входе ЕOM, и соответственно постоянной интенсивности в импульсе, и при постоянной средней интенсивности. В последнем случае среднюю интенсивность пришлось ограничить десятью процентами от уровня максимально достижимой интенсивности накачки. Как отмечалось ранее, крутизна сигнала определяется амплитудой первой гармоники спектра модулирующего сигнала; соответственно при постоянной интенсивности в импульсе крутизна (рис. 8, $b$ ) хорошо описывается синусоидальной зависимостью $\sim \sin \left(\pi \cdot k_{f}\right)$, достигая максимума при 50\% скважности. Ширина в этом режиме (рис. 8,a) очень слабо зависит от $k_{f}$ и снижается в минимуме на $12 \%$. Положение минимума $\left(k_{f} \approx 40 \%\right)$ определяется компромиссом между двумя факторами эффективностью накачки, максимум которой достигается при $k_{f}=50 \%$, и узостью веера моментов. 

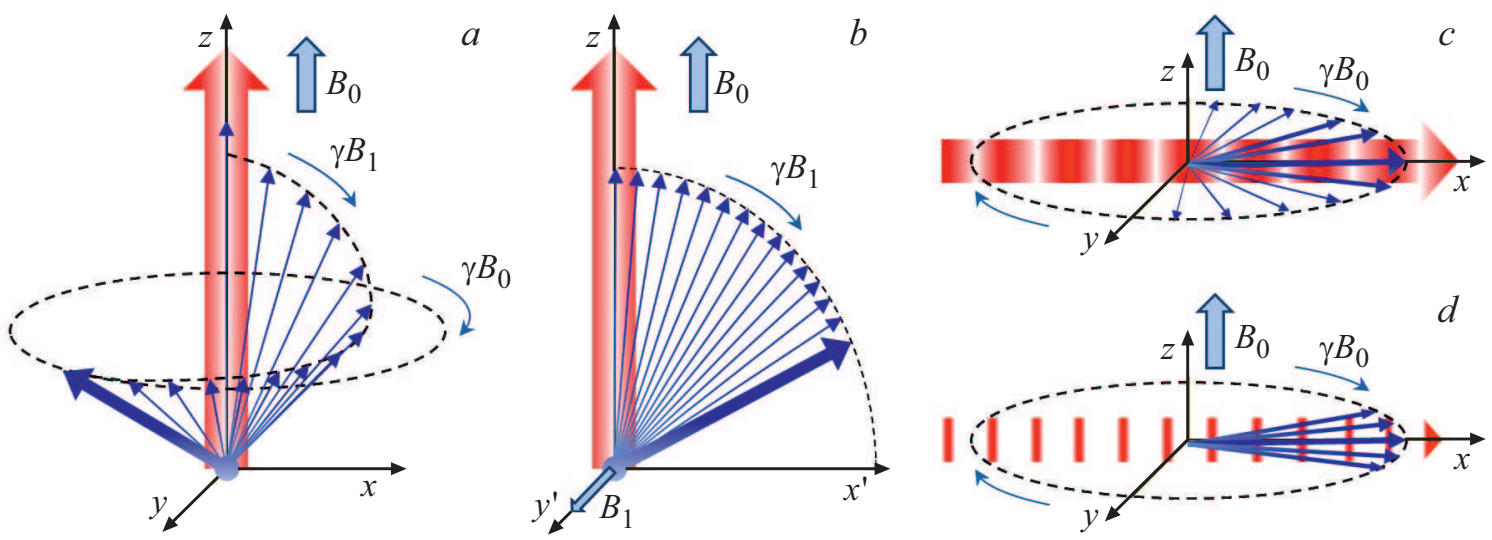

Рис. 6. Оптическая накачка в „классической“ схеме $(a, b)$ и в схеме Белла-Блюма $(c, d)$. $a, c, d$ - лабораторная система координат, $b$ - система координат, вращающаяся с частотой радиополя, резонансного магнитному переходу.
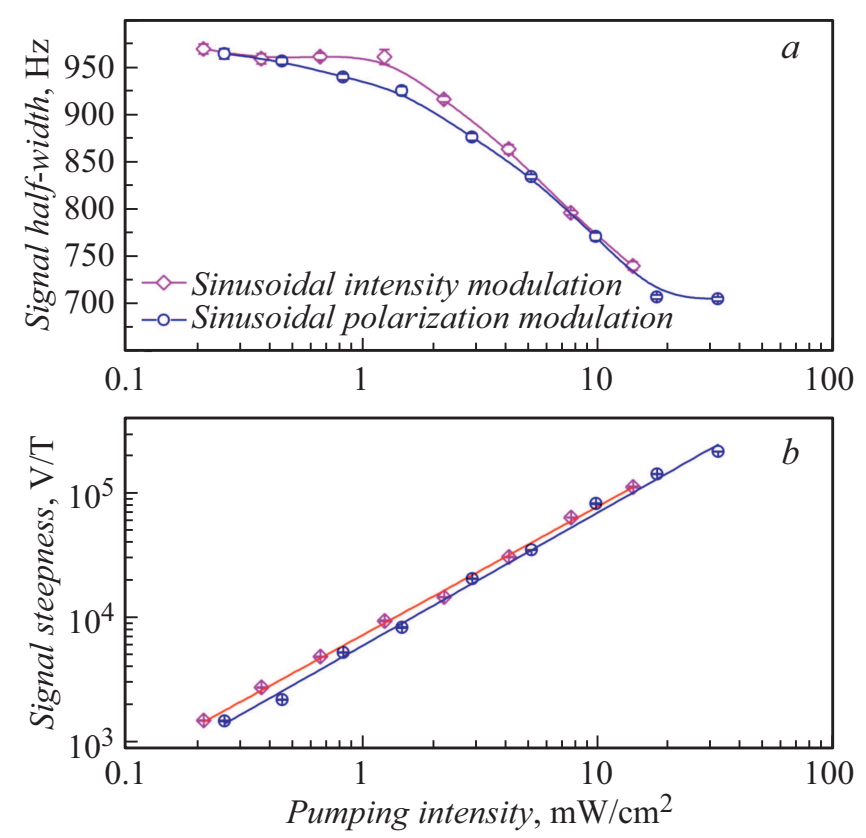

Рис. 7. Сравнение зависимостей полуширины линии MP $(a)$ и крутизны линии MP $(b)$ от интенсивности излучения накачки при синусоидальной модуляции интенсивности и поляризации и при слабой $(0.5 \mathrm{~mW})$ мощности пробного луча.

То, что максимально узкое распределение моментов в фазовом пространстве достигается при минимальном коэффициенте заполнения $k_{f}$, следует из зависимости ширины МР от $k_{f}$ при постоянной средней интенсивности (рис. $8, a)-$ с увеличением коэффициента заполнения ширина МР начинает расти, достигая максимума при $k_{f} \approx 50 \%$. Отметим, что при больших коэффициентах заполнения $\left(k_{f}=80-90 \%\right)$, при которых крутизна падает примерно на порядок, и, следовательно, „вытянутое“ состояние не реализуется, ширина резонанса тоже снижается. Вероятнее всего, этот эффект связан с артефактами, искажающими форму линии при малых амплитудах МР.
Из приведенных выше данных можно сделать вывод, что оптимальный способ модуляции накачки в двухлучевой схеме Белла-Блюма - это импульсная накачка с малым коэффициентом заполнения (высокой скважностью), и интенсивностью достаточно высокой для того, чтобы обеспечить среднюю по времени интенсивность накачки на уровне, достаточном для существенного подавления спин-обменного уширения. Так, если в „классической“ схеме для максимального подавления светового уширения в условиях нашего эксперимента необходима интенсивность $20-40 \mathrm{~mW} / \mathrm{cm}^{2}$ (рис. 5), то в схеме Белла-Блюма при импульсной модуляции интен-
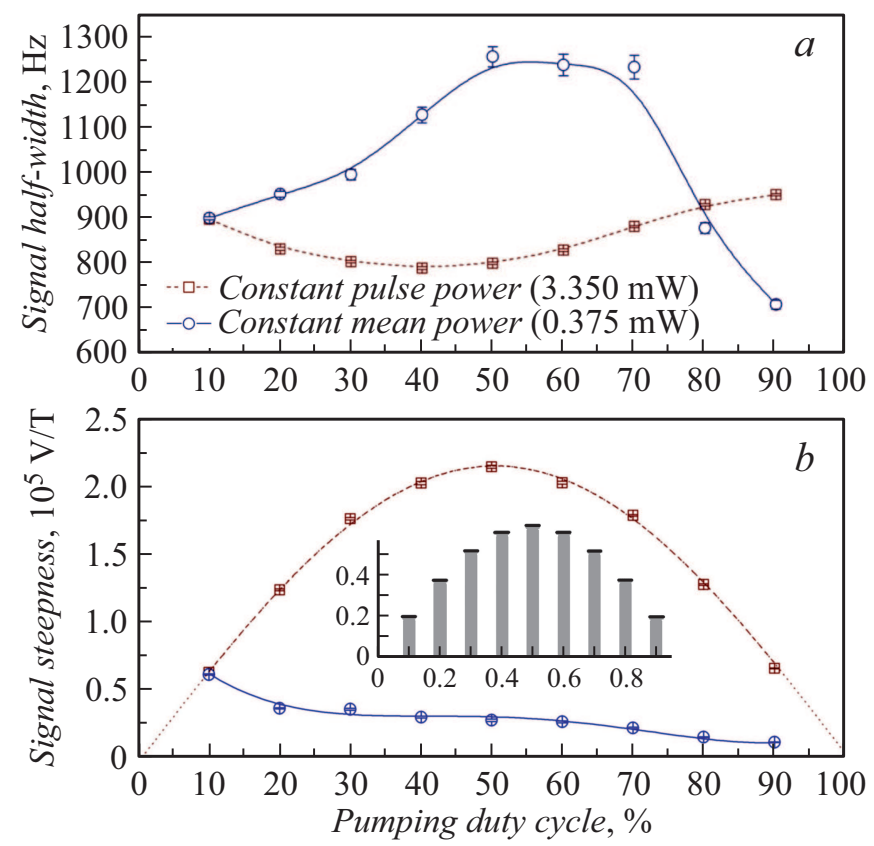

Рис. 8. Полуширина линии MP $(a)$ и крутизна линии MP $(b)$ при изменении коэффициента заполнения $k_{f}$ прямоугольной модуляции интенсивности накачки и при слабой $(0.5 \mathrm{~mW})$ мощности пробного луча. На врезке - амплитуды первых гармоник спектра модулирующего сигнала в зависимости от $k_{f}$. 
сивности с коэффициентом заполнения $10 \%$ потребуется интенсивность порядка $200-400 \mathrm{~mW} / \mathrm{cm}^{2}$, что при сечении луча $0.25 \mathrm{~cm}^{2}$ соответствует мощности $50-100 \mathrm{~mW}$.

Требуемая мощность лазера может быть снижена вдвое, если осуществлять импульсную прямоугольную модуляцию интенсивности на удвоенной ларморовской частоте, переключая в каждом следующем импульсе знак круговой поляризации накачки, и тем самым подкачивая моменты дважды за период прецессии. Использование такого способа модуляции потребует использования двух ЕОМ, зато позволит избежать паразитной засветки фотоприемника модулированным на ларморовской частоте излучением накачки.

\section{Заключение}

Проведено экспериментальное сравнение эффективности оптической накачки в „классической“ двухлучевой схеме наблюдения магнитного резонанса и в различных вариантах схемы Белла-Блюма, использующей модуляцию параметров поперечной накачки. Измерения производились в компактной газовой ячейке при сильной лазерной накачке, обеспечивающей значительное подавление спин-обменного уширения. Показано, что схема Белла-Блюма, использующая модуляцию интенсивности либо поляризации накачки, имеет преимущество в задачах, не требующих абсолютной точности, но требующих сочетания чувствительности с высоким быстродействием - например, в системах МЭГ и МРТ сверхслабого поля. Для дальнейшего повышения крутизны сигнала магнитного резонанса в схеме Белла-Блюма необходимо применять специальные методы модуляции излучения накачки, а именно - сочетание импульсной модуляции интенсивности с высокой скважностью с модуляцией поляризации при одновременном увеличении мощности накачки.

\section{Конфликт интересов}

Авторы заявляют, что у них нет конфликта интересов.

\section{Список литературы}

[1] D. Budker, M. Romalis. Nature Phys., 3, 227 (2007). DOI: $10.1038 /$ nphys 566

[2] M. Hämälinen, R. Hari, R.J. Ilmoniemi, J. Knuutila, O.V. Lounasmaa. Rev. Mod. Phys., 65 (2), 413 (1993). DOI: 10.1103/RevModPhys.65.413

[3] A.M. Coffey, M.L. Truong, E.Y. Chekmenev. J. Magn. Reson. 237, 169 (2013). DOI: 10.1016/j.jmr.2013.10.013

[4] W.E. Bell, A.L. Bloom. Phys. Rev. Lett., 6 (6), 280 (1961). DOI: 10.1103/PhysRevLett.6.280

[5] I.K. Kominis, T.W. Kornack, J.C. Allred, M.V. Romalis. Nature, 422, 596 (2003). DOI: 10.1038/nature01484

[6] H.B. Dang, A.C. Maloof, M.V. Romalis. Appl. Phys. Lett., 97 (15), 151110 (2010). DOI: 10.1063/1.3491215
[7] T. Scholtes, V. Schultze, R. IJsselsteijn, S. Woetzel, H.G. Meyer. Phys. Rev. A, 84, 043416 (2011). DOI: $10.1103 /$ PhysRevA.84.043416

[8] V. Schultze, B. Schillig, R. IJsselsteijn, T. Scholtes, S. Woetzel, R. Stolz. Sensors, 17 (3), 561 (2017). DOI: $10.3390 / \mathrm{s} 17030561$

[9] N.D. Bhaskar, J. Camparo, W. Happer, A. Sharma. Phys. Rev. A, 23, 3048 (1981). DOI: 10.1103/PhysRevA.23.3048

[10] А.К. Вершовский, А.С. Пазгалев, М.В. Петренко. Письма в ЖТФ, $46 \quad$ (17), 43 (2020). DOI: 10.21883/PJTF.2020.17.49894.18340 [A.K. Vershovskii, A.S. Pazgalev, M.V. Petrenko, Tech. Phys. Lett., 46 (9), 877 (2020). DOI: 10.1134/S1063785020090126]

[11] Е.Н. Попов, В.А. Бобрикова, С.П. Воскобойников, К.А. Баранцев, С.М. Устинов, А.Н. Литвинов, А.К. Вершовский, С.П. Дмитриев, В.А. Картошкин, А.С. Пазгалев, М.В. Петренко. Письма в ЖЭТФ, 108 (8), 543 (2018). DOI: 10.1134/S0370274X18200043 [E.N. Popov, V.A. Bobrikova, S.P. Voskoboinikov, K.A. Barantsev, S.M. Ustinov, A.N. Litvinov, A.K. Vershovskii, S.P. Dmitriev, V.A. Kartoshkin, A.S. Pazgalev, M.V. Petrenko. JETP Lett., 108, 513 (2018). DOI: 10.1134/S0021364018200122]

[12] I. Fescenko, P. Knowles, A. Weis, E. Breschi. Opt. Express, 21 (13), 15121 (2013). DOI: 10.1364/OE.21.015121

[13] А.К. Вершовский, С.П. Дмитриев, Г.Г. Козлов, А.C. Пазгалев, М.В. Петренко. ЖТФ, 90 (8), 1243 (2020). DOI: 10.21883/JTF.2020.08.49533.43819 [A.K. Vershovskii, S.P. Dmitriev, G.G. Kozlov, A.S. Pazgalev, M.V. Petrenko, Tech. Phys., 65, 1193 (2020). DOI: 10.1134/S1063784220080204] 